\title{
Evaluation of Position Resolution for a Prototype Whole-Body PET Detector Based on Suppressing Backgrounds by Compton Scattering
}

\author{
Kento FUJIHARA, Yusaku EMOTO, Hiroshi ITO, Naomi KANEKO, Hideyuki KAWAI, \\ Atsushi KOBAYASHI, and Takahiro MIZUNO
}

\begin{abstract}
Existing PET (Positron Emission Tomography) systems make clear images in demonstration (measuring small PET reagent in pure water), however images in real diagnosis become unclear. The authors suspected that this problem was caused by Compton scattering in a detector. When PET systems observe plural photomultiplier tube outputs, an original emission point is regarded as centroid of the outputs. However, even if plural emission in Compton scattering occur, these systems calculate original point in the same way as single emission. Therefore, the authors considered that rejecting Compton scattering events makes PET systems much better, and made prototype counter. Main components of the prototype counter are plate-like high-growth-rate (HGR) La-GPS scintillators and wavelength shifting fibers (WLSF). HGR crystals grow 10 times as fast as a mono-crystal (a normal mono-crystal grows at $2-3 \mathbf{m m}$ an hour). Thus, it includes microbubble and its transparency get worth. Consequently, HGR crystals usually are not used in radiation measuring instruments. However, this time they are used on the purpose. Because of their low transparency, scintillation lights come out right above and right under of emission position. Therefore, Compton scattering events is rejected easily. The prototype detector has an effective area of 300 by 300 square $\mathbf{m m}$. The detector consists of 24 layers. One layer consists of HGR La-GPS scintillator of $1 \mathrm{~mm}$ thickness. Top and bottom surface of scintillator were covered by dual sheets of WLSF with a diameter of 0.2 $\mathrm{mm}$. Sheets of WLSF on top and bottom of the scintillator make a right angle with each other, and measure $X$ - and $Y$-components. Z-component is measured by difference of WLSF outputs between top and bottom. If plural layers output signals, this counter regards the event as Compton scattering event, and reject the event. Even if only a layer output signals, the event is rejected when number output signals from WLSF is more than 1.5 times of single emission. Material cost of this system is, $0.2 \mathrm{M}$ \$ for HGR La-GPS, 0.03MS for WLSF, 0.03MS for 600 units of 6 by $6 \mathrm{~mm}$ SiPM's, $0.12 \mathrm{M} \$$ for 12000 units of 1 by $1 \mathrm{~mm}$ SiPM's, and 0.09M\$ for 1800 channel of signal readout circuits. Considering total cost, price of this PET will be set 1M\$ or less. This idea was confirmed with numerical simulation and experimentation. In experimentation, position resolution in photoelectric absorption was $0.2 \mathrm{~mm}$, and minimum distance that this detector could recognize plural emission in Compton scattering was $1 \mathrm{~mm}$. In parallel, three kinds of model were made: a prototype detector, all the signals readout method, and resistance delay method. Simulation setting was 2 $\mathrm{MBq} / \mathrm{L}$ in normal tissue and $10 \mathrm{MBq} / \mathrm{L}$ in cancer. As a result of simulation, a prototype detector identified $3 \mathrm{~mm}$ cancer, however the others made unclear image and was not able to identified cancer. That is to say, the prototype detector is able to reject Compton scattering events and inexpensive. Therefore, whole-body PET system with this detector must diagnose cancer with a diameter of $3 \mathrm{~mm}$ or more and be priced 1MS or less
\end{abstract}

This manuscript was submitted on June 1, 2017.

K. FUJIHARA is with the Graduate School of Science and technology, Chiba University, Chiba, 263-8522 Japan (e-mail: kento_fujihara@hepburn.s.chiba-u.ac.jp).
Index Terms-Whole-body PET, La-GPS, Wavelength sifting fiber

\section{INTRODUCTION}

E XISTING PET systems have a problem caused by

Compton scattering. In scintillators, scattering occur 4 times as much as photoelectric absorption with $0.511-\mathrm{MeV}$ gamma ray. Even if they have high position resolution, misidentifying plural emissions as a single emission makes reconstructed image unclear. Based on this problem, we are developing a lower cost popular type PET.

\section{METHOD}

Main components of the system are wavelength-shifting fibers (WLSF) and platelike high-growth-rate (HGR) La-GPS ( $\left[\mathrm{Ce}_{0.01} \mathrm{La}_{0.24} \mathrm{Gd}_{0.75}\right]_{2} \mathrm{Si}_{2} \mathrm{O}_{7}$ ) scintillators.

WLSF is an optical fiber kneaded fluorescent material into. It can transmit light from the side of a fiber.

HGR crystals are produced at lower cost and have low transparency because of microbubbles. This is useful for reducing cost and identifying scattering. In this system, emission positions are measured by WLSFs' sheets on top and bottom of scintillators. If transparency of scintillator is moderately low, scintillation lights don't spread in scintillator; nevertheless, sufficient quantity of light is ensured.

The size of detection area is $300 \mathrm{~mm} \times 300 \mathrm{~mm}$. The detector consists of some scintillators. Top and bottom surface of scintillator were covered by dual sheets of WLSF with a diameter of $0.2 \mathrm{~mm}$. Sheets of WLSF on top and bottom of the scintillator make a right angle with each other, and measure $X$ and Y-components. Z-component is measured by the layer number and difference of the number of WLSFs outputting signal between top and bottom. If plural layers output signals, this counter identifies the event as scattering event. Even if only a layer output signals, the event is identified as a scattering event when the number of WLSFs outputting signals is 1.5 times more than that of a single emission. When the event is identified as scattering event, this system regard nearest emission point to body as the first emission point. 


\section{EXPERIMENT AND SIMULATION}

In this study, two preliminary tests were performed for developing the PET detector by using a ${ }^{22} \mathrm{Na}$ sources (Fig. 1-2), where the consistency was demonstrated by using a numerical simulation, Monte Carlo code: GEANT4 (Fig. 3).

In experiment 1 , we measured amount of luminescence from La-GPS (1 mm thickness, top surface of scintillator is covered by sheets of WLSF with a diameter of $0.2 \mathrm{~mm}$ ) with ${ }^{22} \mathrm{Na}$ gamma-ray source. The number of sheets is from 1 to 6 . On each number of sheets, we measured 3 runs and calculated the average. (One run includes 10000 events.)

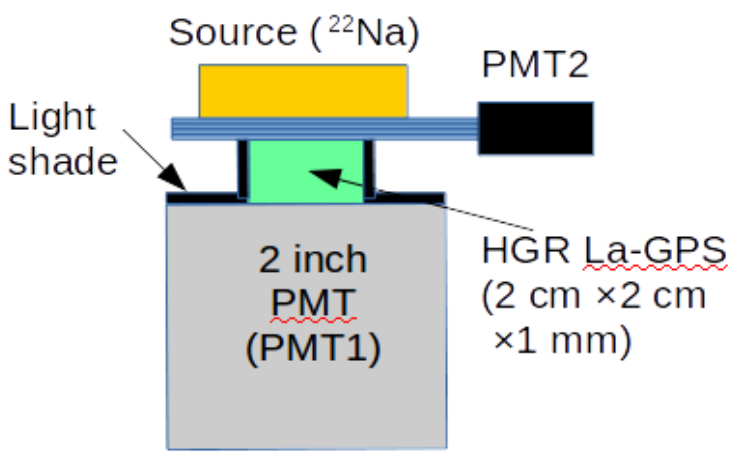

Fig. 1. This is the setting of experiment 1. PMT1 is the trigger detector

In experiment 2, we measured position resolution of this system. PMT0 and PMT1 are event triggers. Scintillator on PMT1 has small solid angle, therefore gamma-rays from ${ }^{22} \mathrm{Na}$ become narrow. The fiber sheet has 48 fibers and each of them is connected to SiPM. Signals from fibers are analyzed with centroid formula given by

$$
\operatorname{pos}_{\text {rec }}=\frac{\sum_{i} n_{\text {p.e.,i }} \times\{10.0 / 48 \times(i+0.5)\}}{\sum_{i} n_{p . e ., i}} .
$$

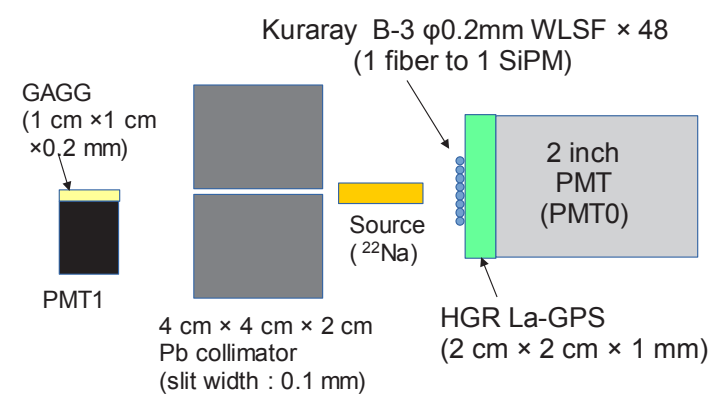

Fig. 2. This is the setting of experiment 2. 511-keV gamma-ray hits center of PMT0.

In the simulation, six detectors surround human $(30 \mathrm{~cm}$ in diameter and filled with water) like a hexagon. One detector has

TABLE I: PARAMETERS OF SIMULATION

\begin{tabular}{cc}
\hline Parameter & Quantity \\
\hline $\begin{array}{c}\text { Radioactivity concentration } \\
\text { (normal tissue) }\end{array}$ & $2 \mathrm{Mbq} / \mathrm{kg}$ \\
\hline $\begin{array}{c}\text { Radioactivity concentration } \\
\text { (cancer) }\end{array}$ & $10 \mathrm{Mbq} / \mathrm{kg}$ \\
\hline Width resolution & $1 \mathrm{~mm}$ \\
\hline Depth resolution & $1 \mathrm{~mm}$ \\
\hline Energy resolution & $13.4 \%(\sigma)$ \\
\hline Time resolution & no error \\
\hline
\end{tabular}

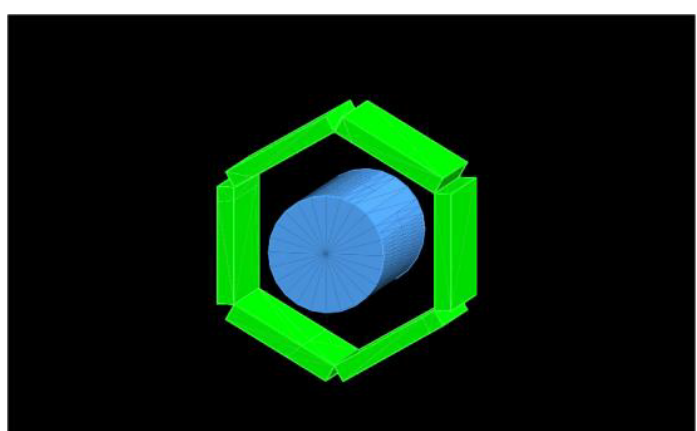

Fig. 3. This is Overall view of simulation in GEANT4. Green blocks are LaGPS scintillators, and a blue tube is human.

$300 \mathrm{~mm}$ width and $24 \mathrm{~mm}$ thickness. If distance between two reaction point is longer than $1 \mathrm{~mm}$, the event is regarded as a scattering event.

\section{RESUlts}

In experiment 1, detector with HGR La-GPS and WLSF (B3 , Kuraray) measured $22.3 \pm 5.96$ photoelectrons (p.e.). in 460$560 \mathrm{keV}$ and $14.1 \pm 5.58$ p.e. in $290-390 \mathrm{keV}$ from single-face, single-end (Fig. 4-6). This is better value than detector with normal La-GPS. By light output from La-GPS and refractive indexes of La-GPS and WLSFs, efficiency of WLSFs is

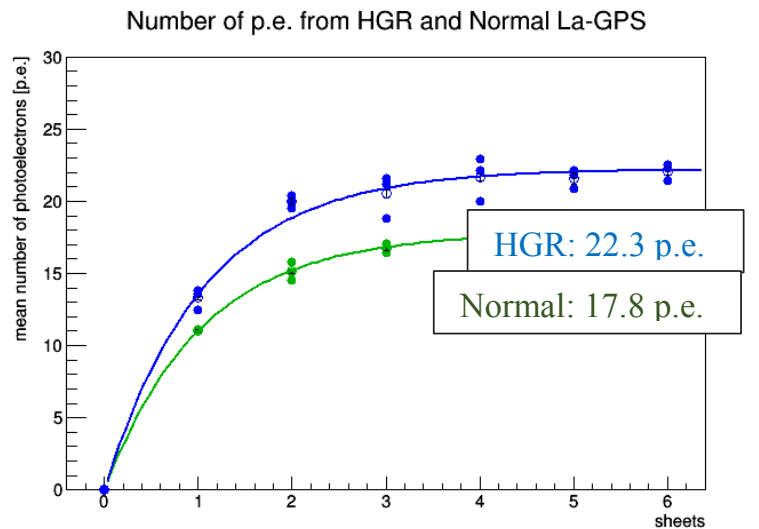

Fig. 4. This is a graph of relationship between the number of WLSF sheets and the number of p.e. in $511-\mathrm{keV}$ gamma rays.
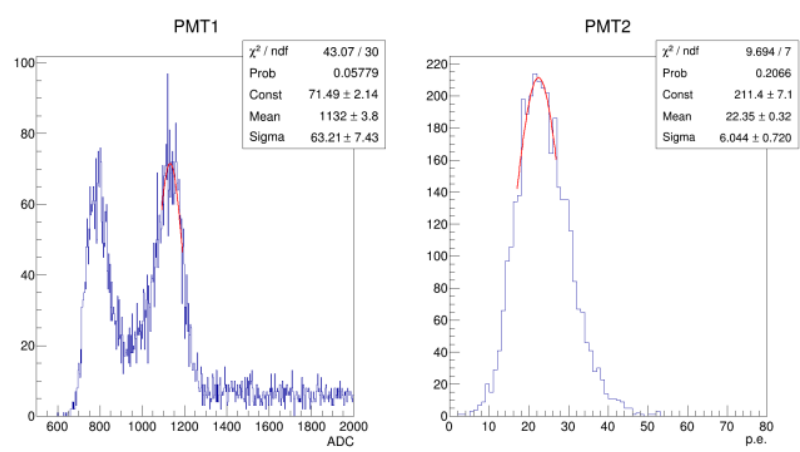

Fig. 5. This is a graph of the number of p.e. in $460-560 \mathrm{keV}$ gamma rays with 6 WLSF sheets.

estimated to be about $4.07 \%$. In addition, we estimated 88 photoelectrons and $36 \%$ resolution in $511-\mathrm{keV}$ from both-faces, both-ends readout. 

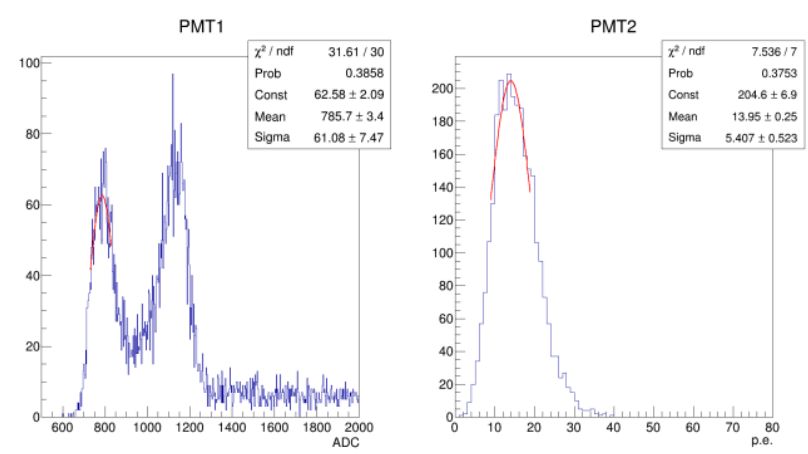

Fig. 6. This is a graph of the number of p.e. in $290-390 \mathrm{keV}$ gamma rays with 6 WLSF sheets.

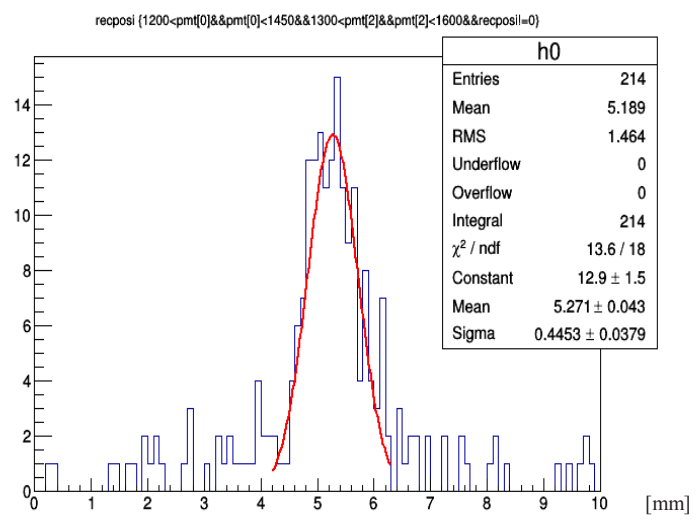

Fig. 7. This is a reconstructed image of the simulation. Central part of histogram is the cancer area.

In experiment 2, position resolution is $1.04 \mathrm{~mm}$ (FWHM) with $1 \mathrm{~mm}$ thickness La-GPS (Fig. 7.).

In simulation, background level is $25.4(\sigma=5.04)$ and cancer level is 17.1. Cancer level is more than $2 \sigma$, therefore cancer is visible (Fig. 8-9). If the criterion is set that 21 voxels in $3 \mathrm{~mm}$

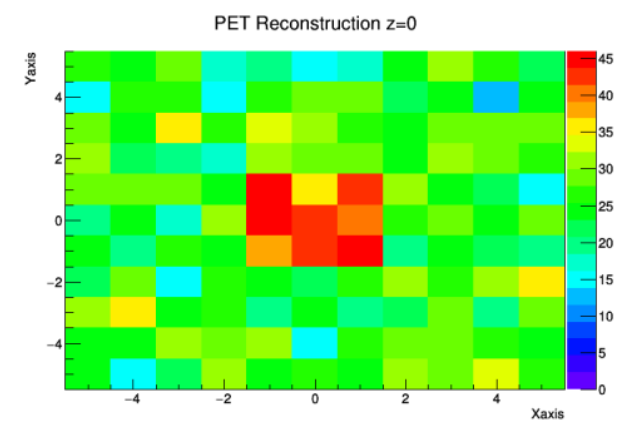

Fig. 8. This is a reconstructed image of the simulation. Central part of histogram is the cancer area.

by $3 \mathrm{~mm}$ by $3 \mathrm{~mm}$ area (27 voxels) are more than $2 \sigma$ from background level, misidentifying probability is $1.55 \times 10^{-35}$, and missing probability is $1.04 \times 10^{-6}$.

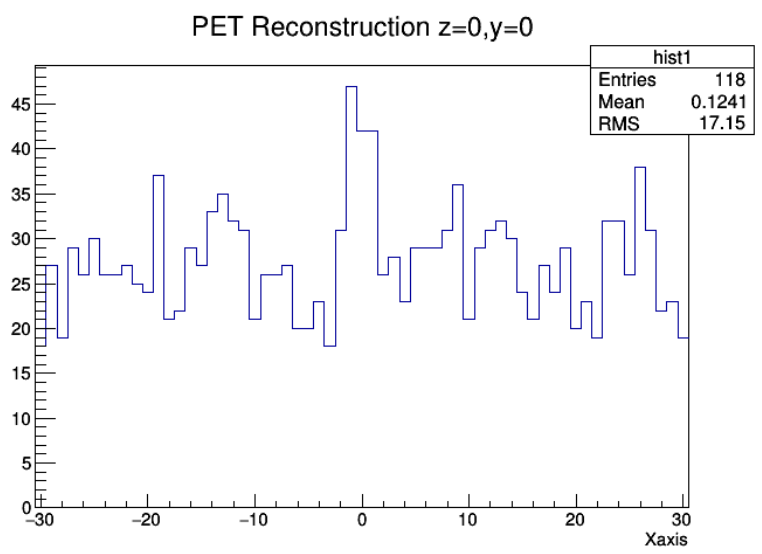

Fig. 9. This is a single dimension reconstructed image of the simulation. Cancer level from background is 17.

\section{DISCUSSION}

In terms of intensity and position resolution, HGR scintillator has sufficient ability. Linearity of number of p.e. is confirmed; Therefore, the number of p.e. with WLSFs in $460-560 \mathrm{keV}$ is about 1.5 times as it in $290-390 \mathrm{keV}$. Value of mean - HWHM in 460-560 keV is 74.1 and value of mean + HWHM in 290-390 $\mathrm{keV}$ is 69.5. Therefore, this detector can distinguish between absorption peak and Compton edge. Position resolution is 1.04 $\mathrm{mm}$. In the simulation reflected experiment results, $3 \mathrm{~mm}$ cancer is visible. This means that the system is feasible and can reduce the cost by HGR scintillators. However, correlation between growth late of HGR La-GPS and transparency is an important subject. We considered that this is closely related to optimal thickness of scintillators, definitive cost and position resolution.

\section{CONCLUSiON}

PET's problem about resolution arise from Compton scattering. Therefore, identifying them makes PET systems much better. According to experiment and simulation, this detector is sufficient for our plan.

\section{REFERENCES}

[1] Berger M J; Hubbell J H; Seltzer S M; Chang J; Coursey J S; Sukumar R; Zucker D S; Olsen K: NIST XCOM: Photon Cross Section Database http://physics.nist.gov/PhysRefData/Xcom/html/xcom1.html (retrieved on the 8th of May 2017) 